\title{
Neuromagnetic Localization of CMV Generators Using Incomplete and Full-Head Biomagnetometer
}

\author{
J . Dammers*,† and A. A. I oannidesł \\ *Institute of Medicine Research CentreJ ülich, 52425 J ülich, Germany; †Department of Physics, Open University, Milton Keynes MK6 7AA, \\ United Kingdom; and łLaboratory for Human Brain Dynamics, Brain Science Institute, RIKEN, Wako-shi 351-01, J apan
}

Received May 19, 1999

Contingent magnetic variation (CMV) data were recorded in three healthy male subjects using a $2 \times 37$ biomagnetometer system. The experiment was repeated for one of the subjects using a 151 whole-head biomagnetometer; the same auditory GO/NOGO choice reaction time paradigm as in the first experiment was used, extended to include repetitions of identical runs and additional control conditions. Magnetic field tomography was applied to the averaged data of each subject, for each run and condition (e.g., GO/NOGO). An independent estimate of the current density in the brain was obtained every few milliseconds. The slow components were emphasized by integrating the square of the current density vector, pixel by pixel, revealing in each subject activity in the auditory cortex, sensorimotor cortex, inferior prefrontal area, and posterior inferior parietal area. The intersubject variability was large, but looking across subjects the auditory and sensorimotor cortex (which were best covered by the two probes) were consistently identified in each subject as contributing to the generation of the early and late slow CMV components. These findings were confirmed by the whole-head single-subject experiment, in which slow activity was also identified in the supplementary motor area (SMA) and posterior cingulate cortex (PCC), areas very likely missed in the first experiment because of the limited view of the twin system. The PCC and particularly the SMA activations were substantially reduced when identical runs were repeated. 2000 Academic Press

\section{INTRODUCTION}

Although the first report about contingent negative variation (CNV) was published 35 years ago by Walter et al. in 1964 there is still considerable uncertainty regarding the neuronal generators of the CNV. In general, the CNV is a surface-negative slow electrical potential generated in the time interval between a warning (S1) and an imperative (S2) stimulus. The activity during the waiting period between the warning and the imperative stimulus differs depending on whether the subject performs (GO) or withhol ds (NOGO) a movement (Walter et al., 1964). This difference is enhanced when a reward or punishment is given following correct or wrong motor responses, respectively. For many subjects, when the two stimuli are separated by a period of $2 \mathrm{~s}$ or more the CNV separates into at least two slow components. The first slow component is referred to as the early CNV, which begins at 300-500 ms after S1 and lasts usually for about $1 \mathrm{~s}$ or less. The second sl ow component, called the late CNV, begins 1.5 to $2 \mathrm{~s}$ before S2 and has its maximum 200 to $300 \mathrm{~ms}$ prior to S2. Studies with intracranial electrodes, in both man and animals, have demonstrated that multiple cortical and subcortical brain areas are involved in the CNV generation ( $\mathrm{Naka}$ mura et al., 1988; I keda et al., 1995, 1998; Hamano et al., 1997; Lamarche et al., 1995). In these studies, however, only the activity in the very local area where electrodes were placed can be identified.

The magnetic counterpart of the CNV is coined the contingent magnetic variation (CMV). The CMV is a laborious and long experiment, but it was neverthel ess studied even with a single channel (Weinberg et al., 1983) and in numerous recent studies with multichannel probes. It is widely accepted that the CMV generators are widely distributed in the brain, a fact which explains the rather limited success in modeling the CMV using only one or a few point-like generators (Elbert et al., 1994). A notable exception is the study of Basile et al. (1994) in which a specific frontal activation was observed, but in a protocol which relied on working memory task with space and pattern recognition components.

In all our CMV studies, past and present, we have used distributed source analysis, which has been successfully applied before the identification of either focal or distributed activations. In one of our earlier studies (Ioannides et al., 1994) magnetic field tomography (MFT) analysis identified activity in each of the two subjects studied in the sensorimotor, supplementary 
motor area (SMA), and parietal areas. In addition, a clear priming of the left (contralateral) auditory cortex was observed in the GO condition when the auditory stimuli was delivered to the right ear (I oannides et al., 1994). In these earlier studies of the CMV we used a single 37-channel probe and hence at any one session either the left or the right temporal areas or the superior parts of the brain could be adequately covered. We investigated all conditions with a centrofrontal dewar location and just one more placement of the single probe (37 channels) over the left lateral area and with a task involving auditory stimuli delivered to the right ear and movement execution or inhibition with the right index finger. Guided by our earlier CMV results (I oannides et al., 1994; Liu et al., 1996) we have undertaken the current CMV study which is in two parts. In the first part (Experiment 1 ) we will present results from three subjects who have been investigated using the BTi twin probe MAGNES system. Each 37-channel probe was placed over the left or right auditory cortex; with such a sensor placement reliable estimates of activity could be obtained in the left and right auditory and motor cortices and to a lesser extent in the frontal and parietal areas. In the second part (Experiment 2) of the study one of the three subjects (J D) volunteered for a repetition of the CMV experiment using the CTF Omega 151a whole-cortex system. The three-subject study with the twin probe verified and extended the earlier results, while the singlesubject analysis of the whole-head data revealed laterality effects and changes in SMA and posterior cingulate cortex (PCC) activations within the same experimental session.

\section{MATERIAL AND METHODS}

\section{Subjects}

Five healthy male volunteers (mean age $37.2 \pm 8.8$; four right handed and one left handed) participated in the MEG experiment in accordance with the Institutional Committee on Human Research. Informed consent was obtained from each volunteer. The data of two subjects were discarded because of unusual large slowfrequency noise in the laboratory's environment at the time of the experiment. The detailed comparison between low-frequency activity across runs and hemispheres was therefore restricted to three of five subjects. None of the remaining three subjects ( $D B, J D$, RB) had performed the CMV experiment before, and hence a detailed demonstration and time for testing was given to all subjects. Subject J D had been a subject in earlier auditory experiments.

\section{Protocol}

In Experiment 1 four conditions (left/right ear presentation and left/right index finger (no) movement) di- vided the long experiment into four runs with intervening pauses, each run lasting for about $11 \mathrm{~min}$. For convenience we will use a four-label code to distinguish between the four different runs: the first index refers to left/right ear presentation and the second index refers to left/right index finger; for example, the combination LR means left ear presentation and right index finger (no) movement. We presented auditory stimuli in blocked conditions but randomly interleaved $30 \mathrm{GO}$ and 30 NOGO trials within each block (ISI $10.8 \pm 2 \mathrm{~s}$ ), in which $1-\mathrm{kHz}$ tones indicated a NOGO and $2-\mathrm{kHz}$ tones a GO condition. Each tone was delivered at $40 \mathrm{~dB}$ above the individual hearing threshold. The warning tone S1 was $250 \mathrm{~ms}$, and the imperative stimuli S2 was $270 \mathrm{~ms}$. The separation (onset-to-onset) between S1 and S2 was chosen to be 3.5 s to separate out the early and late CMV components. For the GO condition, a rapid button-press response was required in the time window of S2 to avoid a 250-ms unpleasant tone burst of white noise delivered with $90 \mathrm{~dB}$ through the headphones. The subjects were requested to keep their eyes open and to fixate on a cross-hair placed $1 \mathrm{~m}$ away. The same protocol was used for the single-subject Experiment 2, in which the only modification was the number of GO and NOGO trials, which were increased to 50 trials. Only two different conditions ( $L L 1, R R 1$ ) were used but each was repeated once ( $L L 2, R R 2)$. Five basel ine runs were recorded, two before and three after the CMV runs: the first (pre-CMV) and the last (postCMV) runs were noise measurements with no subject in place. The second and penultimate runs were noise measurements with the subject in place, but with no tones delivered and with no task set. At the end of the CMV runs, and before the first of the two post-CMV noise measurements, the same stimulation as in condition $L L$ run was repeated but without any response required from the subject. We remark that Experiment 2 was performed 2.5 years after Experiment 1 , by which time subject J D had participated in five CMV experiments, with the same or very similar protocol as the one used in the two experiments reported here. Any changes in the activity observed with this subject are not therefore attributable to the use of a naive subject or insufficient training.

\section{Data Acquisition}

The MEG signal in Experiment 1 was recorded from each of the three subjects using the BTi MAGNES II system; this consists of two dewars, each housing 37 first-order axial gradiometer channels. The experiment was performed in a magnetically shielded room with lateral placement of either probe over the left and the right side of the head (Figs. 1a and $1 b$ ). The positioning of each probe was repeated until the channels of each probe captured the positive and negative field extrema associated with the auditory M100 peak. The single- 


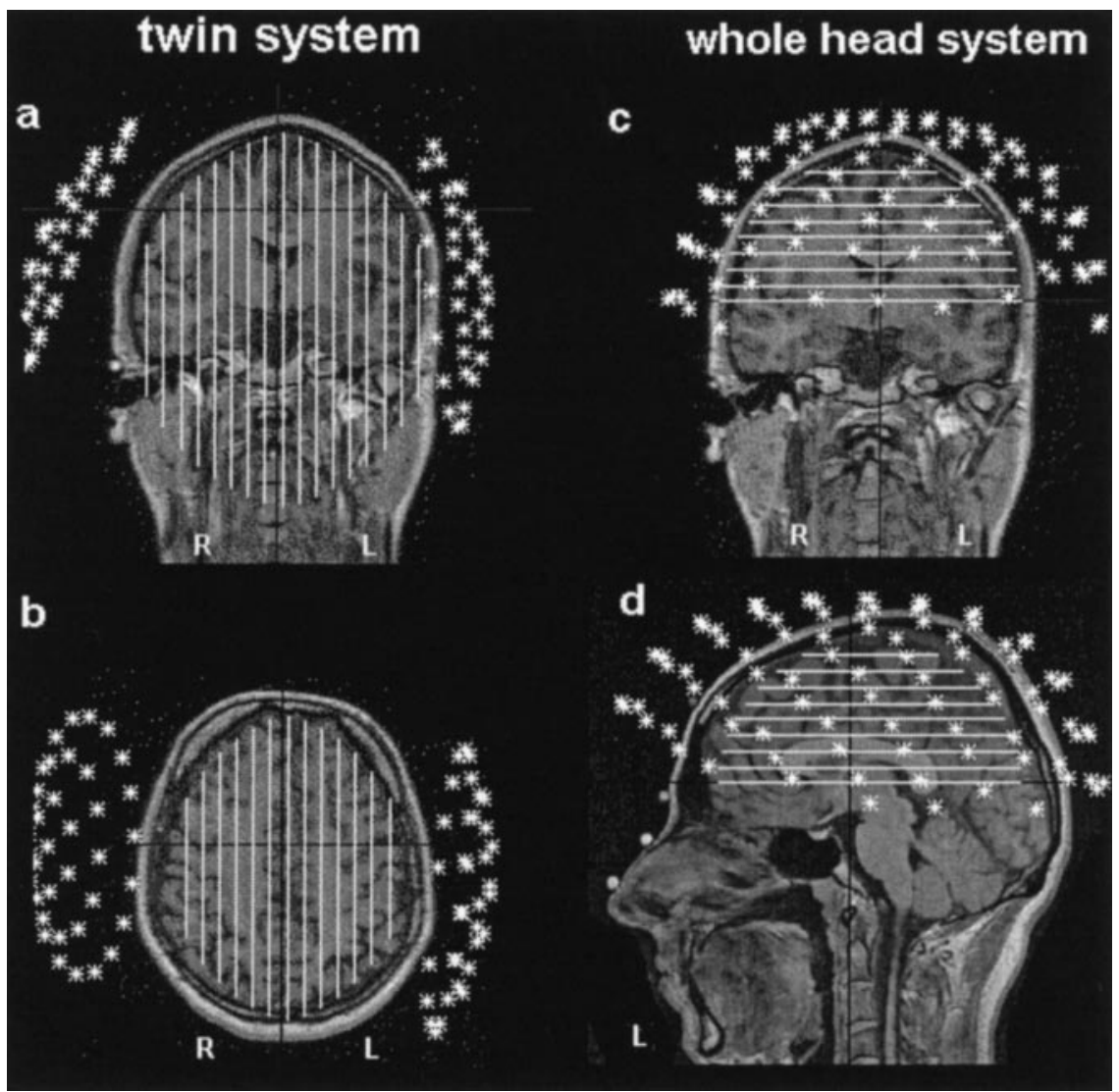

FIG. 1. Channel selection and source space definition. (a and b) The channel layout for Experiment 1 performed with the BTi MAGNES II system and the respective left and right source spaces, used for the MFT reconstructions. Nine sagittal slices are shown for each source space, each appearing as a line in the coronal and axial MRI slices displayed (the deepest slice is common to the left and right source spaces). (c and d) The 90 channels and the top source space used in addition to the lateral source space estimates for the MFT analysis of the helmet MEG data. The coronal and sagittal views are shown, with the nine levels of the top source space cutting the displays at axial planes projected as lines on the display.

subject experiment (Experiment 2) was performed using the CTF Omega 151a whole-cortex system, which consists of a 151 first-order axial gradiometer (Figs. 1c and $1 d$ ). Third-order synthetic gradient formation was used to reduce the low-frequency band in the wholehead MEG data (Cheyne et al., 1995). The neuromagnetic activity was continuously recorded with a sampling rate of $1042 \mathrm{~Hz}$ and a bandwidth from DC to 400 $\mathrm{Hz}$ (Experiment 1) and a sampling rate of $1250 \mathrm{~Hz}$ with a bandwidth from DC to $300 \mathrm{~Hz}$ (Experiment 2). In all experiments, separate EOG, ECG, and EMG channels were continuously recorded and in synchrony with the MEG channels. A fiber-optic finger movement detection device was used to record the finger movement.

\section{Data Analysis}

For further analysis the data were low-pass filtered from DC to $20 \mathrm{~Hz}$ to study the slow components as well as band-pass filtered from 3 to $20 \mathrm{~Hz}$ for the evoked-like activity. After separation between correct GO and NOGO trials, separate averages were constructed time-locked to both the onset of the warning stimulus (tone averages) and the onset of the TTL signal of the buttonpress device (button-press averages). The number of trials that remained for averaging in Experiment 1 were (on average) $28.2( \pm 1.2)$ for subject J D, 29.5 $( \pm 0.6)$ for subject $D B$, and $26.5( \pm 2.3)$ for subject FB, while in Experiment 2 the number of trials was 33 ( \pm 3.4$)$. MFT, which computes the nonsilent primary current density $\mathrm{J}(\mathrm{r}, \mathrm{t})$ was applied to averaged data separately for the left and right hemisphere to extract time courses of activations (I oannides et al., 1990). F or each hemisphere a separate source space Q (i.e., a 3-D region of space in which ${ }^{\mathrm{P}}(r, t)$ was allowed to be non-zero) was defined to be a spherical segment that fitted the entire hemisphere (Figs. $1 \mathrm{a}$ and $1 \mathrm{~b}$ ). For Experiment 2 an additional top source space was used covering brain areas above the anterior and the posterior commissure (Figs. 1c and 1d). Separate computations were performed for each source space. The reconstructed current densities will be displayed on the background anatomy as gray-level intensity $(P(r, t)=$ 
$\mathrm{J}^{\mathrm{P}}(r, t)^{2}{ }^{2}$. Smoothed integrals of intensity, $A(t, \Delta t)$, in a region of interest (ROI), are defined by

$A_{R O I}(t, \Delta t)=\int_{t-\Delta t / 2}^{t+\Delta t / 2} d t^{\prime} \int_{R O I} P\left(r, t^{\prime}\right) e^{-\left(r-r_{0}^{R O I}\right)^{2} / \lambda^{2}} d^{3} r$.

Each ROI is bounded by a spherical shell with center at the point $r_{0}^{\text {ROI }}$; the Gaussian factor controls how points away from the center of the ROI are weighted. In this study we focus on the dynamic of the strongest superficial activations, defined on functional criteria (strongest activity identified by MFT). These functionally defined ROIs were projected onto the individual subject's MRIs. The time course of each ROI was calculated for each subject from separate average signals for each case (LL, RR) computed separately for the GO and NOGO condition as well as for the tone-only case and for the noise runs (averaged in exactly the same way as for the CMV and tone-only presentation cases).

In this study, we will emphasize the slow CNV-type components. These components were difficult to extract because the effectiveness of the passive shielding is greatly reduced, and hence one is more vulnerable to contamination from environmental noise. We will therefore primarily apply MFT analysis to the 0 - to $20-\mathrm{Hz}$ data and further amplify the slow components by integrating the intensity as described by Eq. (1) using a running window of $500 \mathrm{~ms}$ width. For the reconstructions with lateral dewar placement (Experiment 1) we were forced to use the side source spaces, with separate computations for the left and right hemisphere. For the full-head coverage (Experiment 2) we used 90 sensors in each of three separate reconstructions, two with source spaces identical to those used for Experiment 2 and an additional one using the top source space shown in Fig. 1c. Experiment 2 used MFT estimates obtained with the top source space for all areas, except the auditory cortex, for which we will use MFT estimates obtained with the side source spaces.

\section{RESULTS}

As expected we found good agreement in reconstructions obtained from the side and the top source spaces in Experiment 2 when areas were well covered by the sensors in both cases. The evoked-like activity obtained from the 3- to $20-\mathrm{Hz}$ data of Experiment 1 and Experiment 2 reproduced the earlier findings (I oannides et al ., 1994; Liu et al., 1996), in which MFT identified the evoked-like activity in the auditory cortex for all subjects, about 100 ms after the tone onset. I n the present study we will restrict attention to the analysis of slow components.

\section{Experiment 1}

In all subjects slow CMV-like drifts were identified in the DC to $20-\mathrm{Hz}$ filtered neuromagnetic signals recorded from both left and right hemisphere. Figure 2 shows typical examples of GO and NOGO channels; for each subject a channel above the sensorimotor or parietal area was chosen from the tone-onset-averaged GO and NOGO trials of the first CMV run. A clearly distinguishable slow magnetic field change is evident in the GO trials in all three subjects with a maximum field amplitude varying from 340 to 840 fT among the subjects. The subject-to-subject variability was large but whether this was due to differences in the probe positioning or actual differences in regional brain activ-
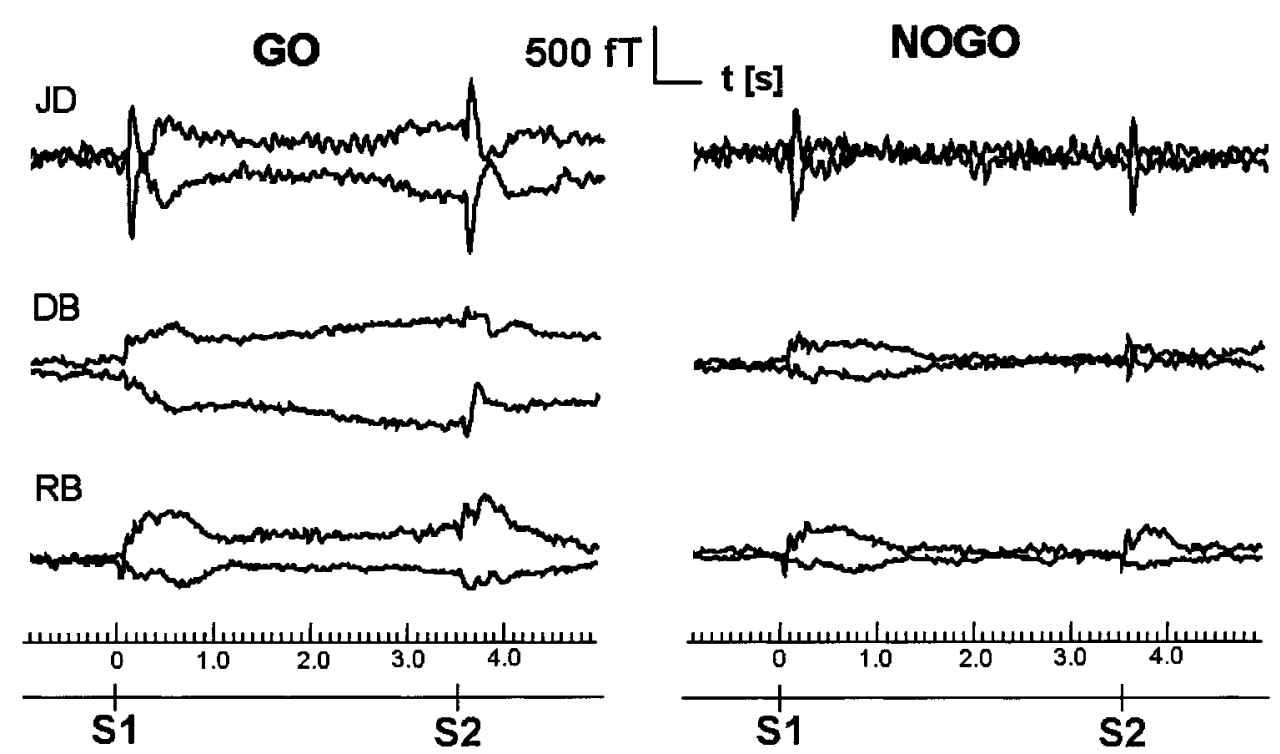

FIG. 2. CMV signal plot for three subjects showing one channel on either hemisphere of the first (tone) averaged CMV run (LL) during GO condition (left) and NOGO condition (right). In each (GO) case the channel with the strongest amplitude is sel ected. 
ity cannot of course be determined by just the morphology of the signals.

MFT analysis of the averaged signals provided the details for the spatiotemporal regional variation (as captured by theaveraging process). Activity was identified in the region of the auditory cortex (AC), motorand sensorimotor cortex (SMC), inferior prefrontal area (IPF), and posterior inferior parietal (PIP) areas (Fig. $5 a)$.

For subject RB, the priming of $A C$ was evident soon after S1 in all four conditions; the AC priming remained high in the GO task. For subjectJ D theAC priming was again evident in all conditions after $\mathrm{S} 1$ but in two conditions ( $R R, R L)$ it was reduced to same level as in the NOGO trace. Figures $3 a$ and $3 b$ show the MFT activations of the left and right $A C$ and SMC obtained from the (tone-onset) average DC to $20-\mathrm{Hz}$ signals of the left-handed subject (DB). Figure 3a (SMC ROI) shows a clear late CMV component in all GO task cases, with onset of about $2 \mathrm{~s}$ before S2. Figure $3 \mathrm{~b}$ shows that for this left-handed subject the AC priming after S1 is present in both the ipsilateral and the contralateral side of the brain. The differentiation between the GO and the NOGO conditions is not as clear-cut as for the SMC, but overall the elevated activity remains high through the late CMV in the GO condition.

While the timing of slow activations in the $A C$ and SMC region were broadly consistent across subjects and runs, the ones from the IPF and PIP were highly variable, especially between subjects. It is unclear whether the variability was due to true intersubject differences, reflecting possibly differences in strategy. The within-subject variability can be studied only by a more detailed examination of one subject and with simultaneous recording over all areas. This we have done in the second part of the study.

Figure 4 shows an example of the distribution of MEG signals over the scalp from the second experiment using the whole-head system. The trace for each sensor is plotted for the average signal from the GO condition of the first LL CMV run. Thefigure shows the characteristic CMV morphology, as al ready demonstrated in Fig. 2 , evident in both hemispheres over the temporoparietal areas, with a stronger slow CMV signal on the contralateral (right) hemisphere. Figure 5 links the two experiments. Figure $5 a$ shows examples of strong instantaneous activations extracted from average MEG data recorded in Experiment 1 (left two columns) and Experiment 2 (right two columns). Activations are displayed at similar but not identical times; the instances displayed were chosen so that the area in question was activated more or less in isolation, at least within the displayed MRI slice. The motor cortex activation is particularly difficult to extract from toneonset-based average MEG signals. Summing the signal from trials time-locked to the onset of the button press

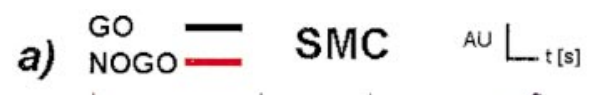

b)
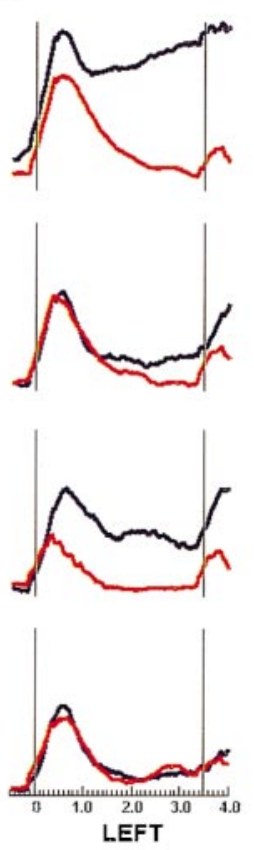

AC
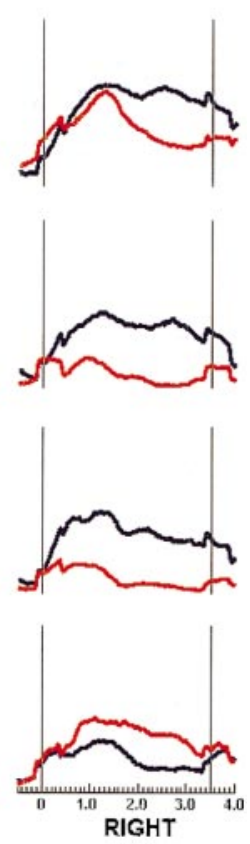

FIG. 3. Time courses of the SMC region (a) and AC region (b) for the left-handed subject DB for the GO (blue line) and NOGO (red line) tone averages of all four conditions. LEFT and RIGHT under the horizontal axes label the source space hemisphere and probe used for the MFT analysis. The first (second) thin vertical line in each graph marks the onset of the warning (imperative) tone. The slow components correspond to integrals of MFT estimates for the modulus of the current density vector over $500 \mathrm{~ms}$. 


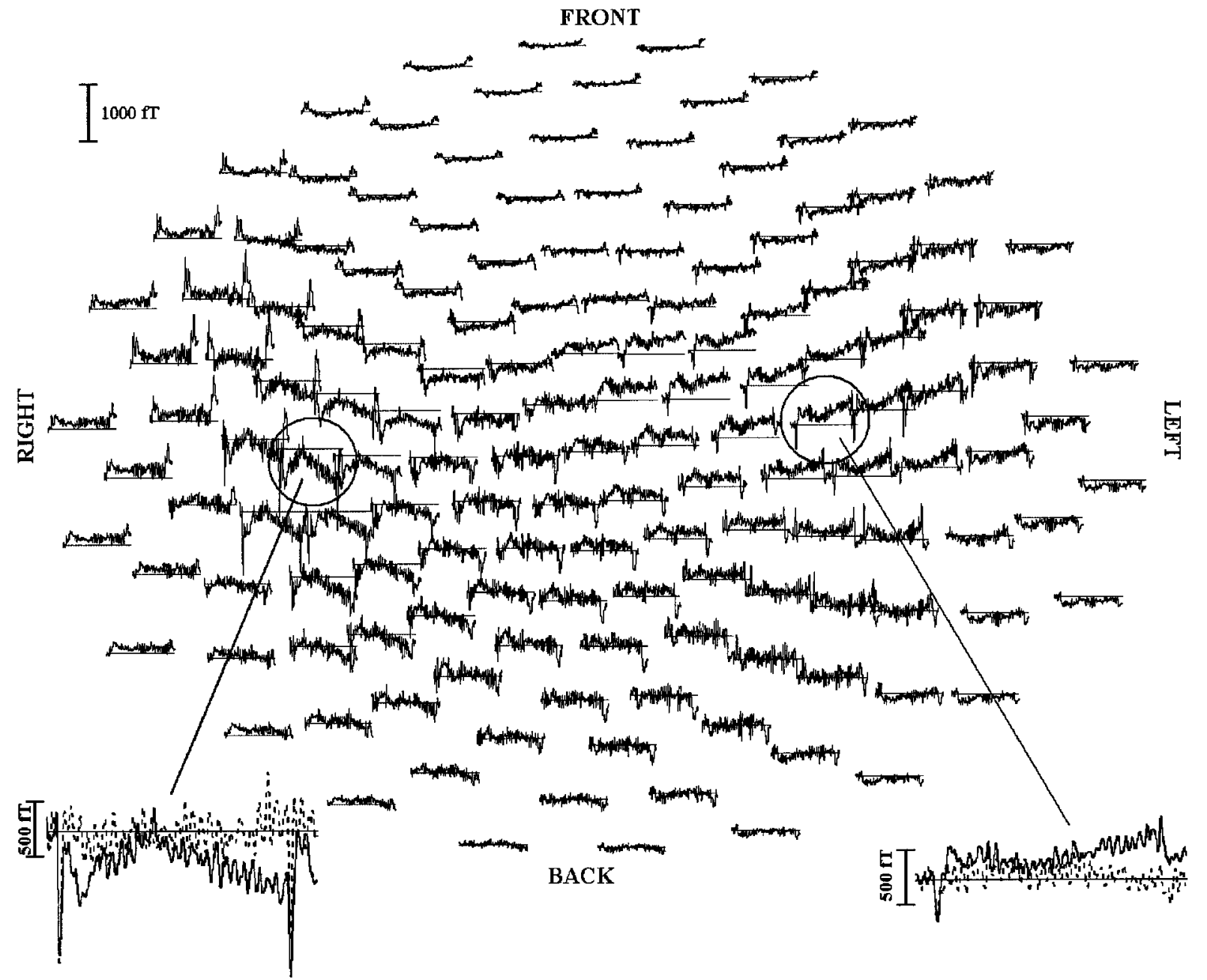

FIG. 4. CMV signal plot of subject J D showing the (tone) average MEG signal (100 ms prestimulus and 4000 ms poststimulus) of the first LL CMV run during GO condition for all 151 sensors. The prominent slow CMV signals are best seen in the temporoparietal areas, with clear enhancement of the contralateral (right) side. One channel on each hemisphere showing the CMV morphol ogy best is highlighted and they are shown in enlargement with the NOGO trace superimposed (dashed).

provides a more appropriate average; the last two images on the right in the second row of Fig. 5a show the SMC MFT estimates from Experiment 2 extracted from the average of single trials filtered in the DC-20 $\mathrm{Hz}$ range and time locked to the onset of the button press. The displayed regional activation corresponds to $40 \mathrm{~ms}$ before the button press. Figure 5b shows two examples of additional areas seen with the helmet data: the SMA and the PCC. As we will see next the activations in these areas are labile, especially at SMA, where they become weaker when the same run is repeated a few minutes or hours later.

\section{Experiment 2}

Figure 6 shows the time courses of the activations in the $A C$ region in the $3-20 \mathrm{~Hz}$ filtered range, for the first $L L$ condition. The auditory cortex activation is evident around $100 \mathrm{~ms}$ after each tone in all conditions irrespec- tive of task (GO, NOGO, or just tone presentation), and in each of these the activation is much higher than the two "noise" runs. Figure 7 shows the time courses of the reconstructed DC to $20-\mathrm{Hz}$ signal (toneaverages) for six areas which have shown up consistently in both experiments. The activations are shown for all CMV conditions ( $L L 1, L L 2, R R 1, R R 2$ ) together with the baseline conditions provided by the tone-only case and the first noise run with subject in place (before the CMV experiment). The activation curves obtained from the AC and SMC ROIs of condition LL1 and RR1 broadly agree with our results from Experiment 1 for the same subject. For the AC, a slow CMV-type component is seen in the right hemisphere, with the early CMV part showing some but not complete differentiation between GO and NOGO conditions. For the SMC, the late component differentiates well between GO and NOGO conditions, especially for the repetition runs. The activ- 


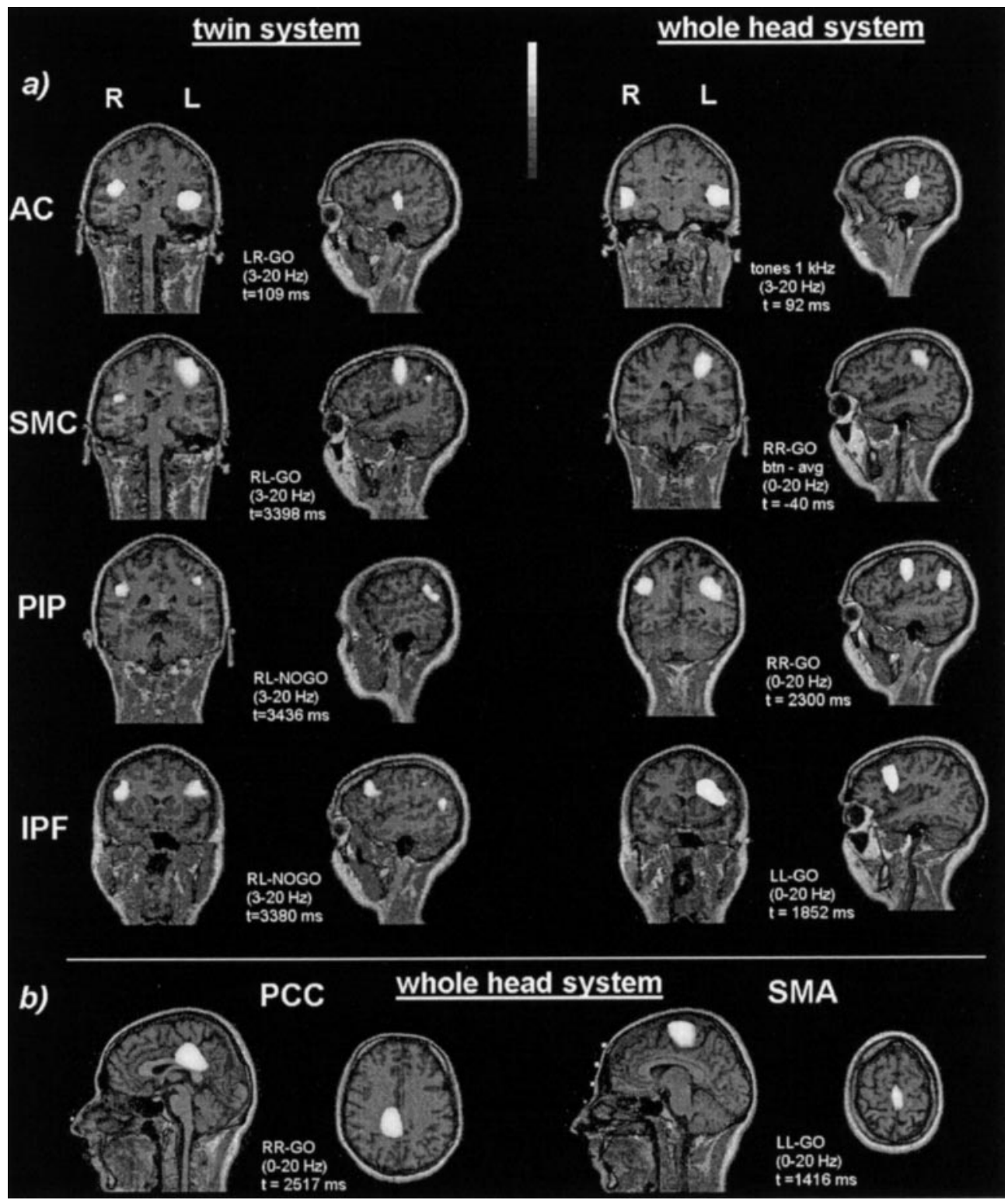

FIG. 5. Instantaneous MFT reconstructions for subject J D, who participated in both experiments. In (a) regional activations are displayed showing in turn from the top: AC, SMC, PIP, and IPF. In each case the activations are superimposed on a coronal and a sagittal slice. The coronal slice is a compromise of the homol ogous sides on the left and right hemispheres. The displayed areas were identified in all four subjects in Experiment 1 and for J D again in Experiment 2. The display for J D in (a) shows on the left (first two images) MFT reconstructions from the twin MAGNES data (Experiment 1) and on the right (last two images) corresponding MFT reconstructions from the helmet data (Experiment 2).The last row (b) shows two new areas, clearly identified only in Experiment 2, the supplementary motor area (SMA) and the posterior cingulate cortex (PCC). All results are obtained from MFT analysis of the instantaneous value of the average signal at the indicated latency. In all images the averages were computed with single trials aligned to the onset of the tone, except for the whole-head sensorimotor cortex activation (second pair of images on the right), in which the average was performed after aligning the single trials with the onset of the button press.

ity extracted from sensorimotor cortex shows a clear late CMV which is strongest on the left hemisphere (subject J D is right handed). The activation extracted from the tone-only case, which was presented in the same way as the $L L$ condition, shows similar responses compared to NOGO traces (Fig. 7).

In the PIP region a late CMV can be observed in GO traces of all conditions, but on the left hemisphere only, while the right PIP ROI shows priming after S1. Both areas SMA and PCC, which were missed by the sensor arrangement of Experiment 1, are primed in all GO cases of both conditions (LL, RR) starting 200-300 ms after S1. In both hemispheres the SMA (GO-) activity remains high after $\mathrm{S} 1$, while a late CMV arises in the 


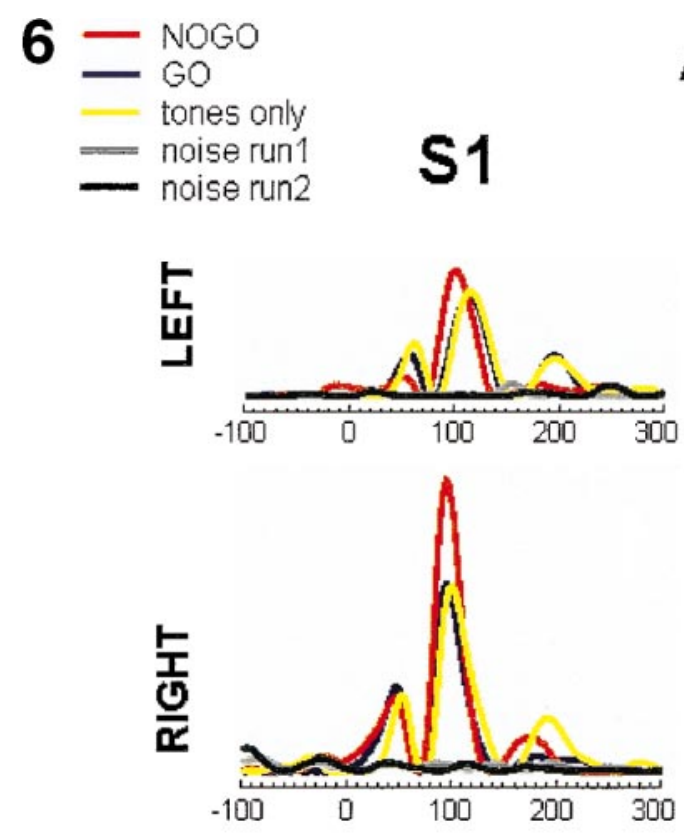

8
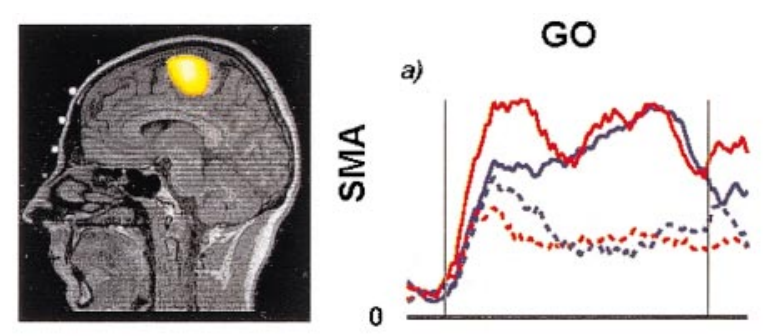

\section{AC}

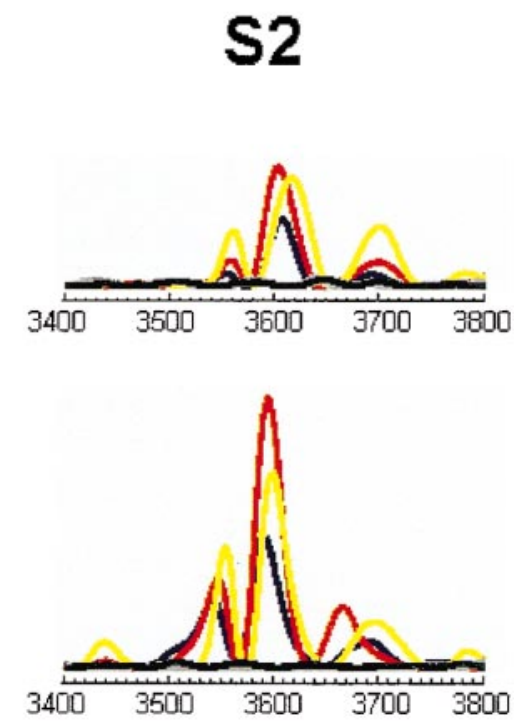

GO-NOGO Difference

b)

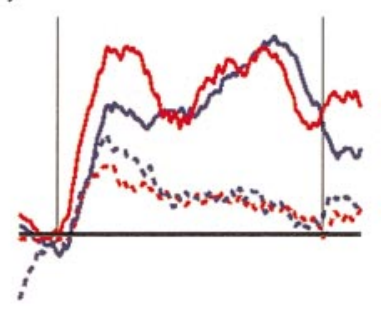
$\begin{array}{ll}R R 1 & \text { 二 } \\ \text { LL1 } & \text { AU } \bigsqcup_{\mathrm{t} / \mathrm{ms}} \\ \text { RR2 } & ----\end{array}$
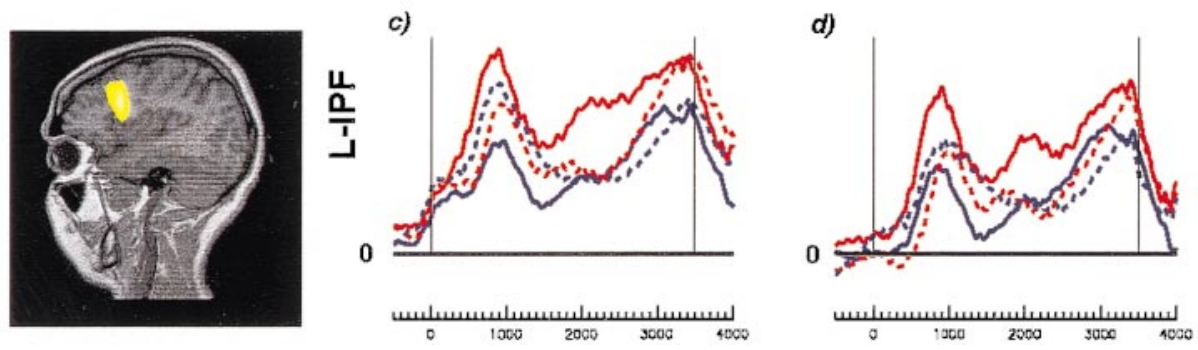

FIG. 6. The evoked-like response in the left and right auditory cortices. Each graph shows the CMV activations (LL 1) as blue (GO) and red (NOGO) lines, together with the tone-only condition (yellow line) and the two noise conditions (gray and black lines). In each case the MFT estimates extracted from the 3- to $20-\mathrm{Hz}$ filtered MEG data are displayed for 400 ms showing the square of the current density J , beginning $100 \mathrm{~ms}$ before the onset of the tone for both S1 and S2 time periods.

FIG. 8. GO case activations for the SMA (a) and LIPF (b) ROI s in comparison to the difference activation between the GO and the NOGO of the same area. In the case of SMA the mean of the left and right SMA activity is displayed. As in previous figures we have integrated the current density modulus over $500 \mathrm{~ms}$. The sagittal MRI slice of the area is displayed together with the activation of the ROI on its right for the four runs.

PCC region with onset of $2 \mathrm{~s}$ before $\mathrm{S} 2$. In some ROIs (AC, SMA, PCC) we observe a reduction of slow activity in runs which have been repeated (LL2, RR2). The strongest reduction is obvious in activation of $\mathrm{GO}$ traces in the region of SMA and PCC. The most dramatic reduction of the GO to NOGO differential in slow activation between $\mathrm{S} 1$ and $\mathrm{S} 2$ is in the SMA. The left I PF area produced the most stable and consistently strong activation differentiating the GO and NOGO conditions. The activity in the left IPF decreased rap- 


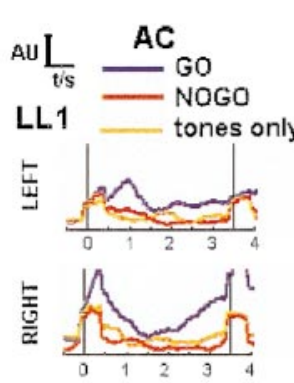

SMC

SMA

PCC

IPF

PIP

\section{LL1 NoGO}
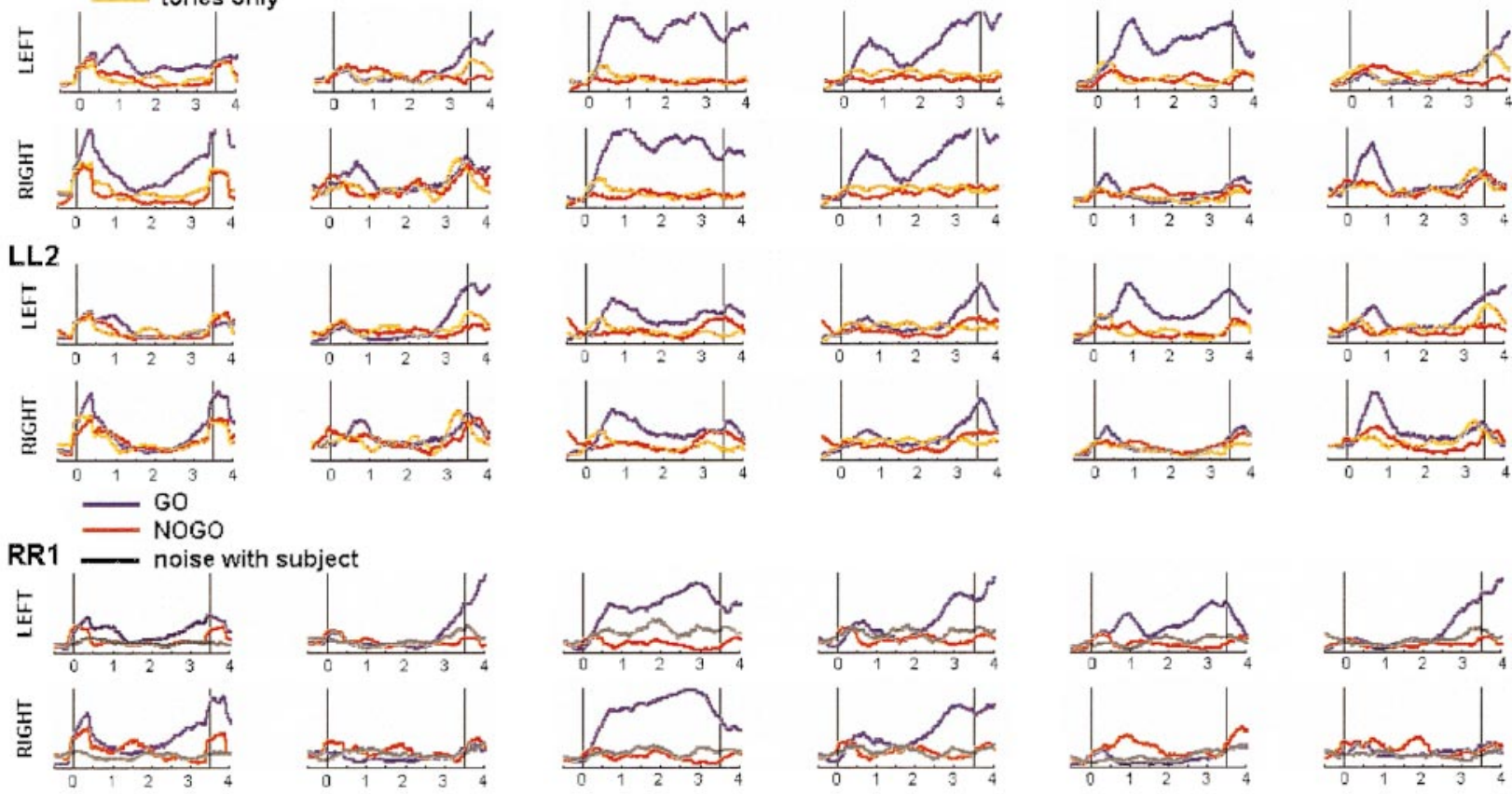

RR2
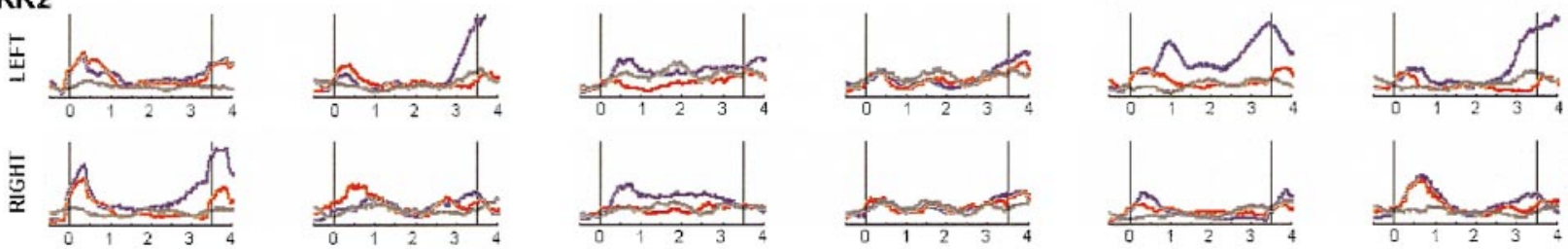

FIG. 7. Time courses of slow regional activations for the AC, SMC, SMA, PCC, IPF, and PIP ROIs, as extracted by MFT analysis of the (tone) average DC to $20-\mathrm{Hz}$ MEG signal from Experiment 2. In each case the instantaneous estimate of the modulus of the current density vector was integrated over 500 ms. In each graph of the top four rows (LL1 and LL2 runs) the traces from the GO (blue) and NOGO (red) are superimposed together with the trace from the tone-only run (yellow), which followed the CMV runs. The SMA and PCC ROI s on the left and right are very similar. In the bottom four rows (RR1 and RR2 runs) the traces from the GO (blue) and NOGO (red) are superimposed together with the trace from the last noise run with subject in place (black), which followed the tone-only run.

idly with the onset of S2. This is the area closest to Sasaki's NOGO area, in terms of both location and function. Figure 8 shows slow activations extracted from GO cases (Figs. 7a and 7c) and the difference between GO and NOGO activations (Figs. $7 \mathrm{~b}$ and $7 \mathrm{~d}$ ) for the SMA and left IPF region, with the trace of different CMV runs superimposed for each area. In the case of SMA the mean of the left and right SMA activity is displayed. The comparison of the GO activity and the difference between GO and NOGO activity shows that in both areas the proportion of the NOGO activity is negligible.

\section{DISCUSSION}

In both experiments we have identified the large slow components that one associates with the CMV protocol. In Experiment 1 the variability across subjects was very large, especially in the wide band filtered data $(0-200 \mathrm{~Hz})$. The slow CMV components were best seen when the activity extracted by MFT from the DC to 20-Hz data was integrated over $500 \mathrm{~ms}$. This integration produced stable and largely reproducible activations. In view of our findings during repetitions of identical runs the considerable variability between subjects which still persisted is unlikely to be just noise, but it may reflect a combination of innate differences in the way function is organized in different individuals, different strategies adopted, and training effects, which even for our extensively trained subject J D were clearly in evidence (e.g., SMA activation across repetitions in Fig. 8). This view is supported by the findings of invasive studies (Lamarche et al., 1995), in which it is found that "the slow potentials recorded in the CNV paradigm were of variable shape, preventing a very systematic classification." Nevertheless, for all 
subjects and runs free of large low-frequency external noise the slow components were consistently identified in the same areas; the variability was primarily in the timing and duration of sl ow CNV-like activations in the different areas. In summary, Experiment 1, with coverage over the left and right hemispheres, identified strong contributions from the auditory cortex and sensorimotor cortex to the early and late CMV, in agreement with our earlier findings (I oannides et al., 1994): In general terms the AC is primed soon after S1 onset as part of the early CMV complex, while the SMC is primed before S2 (as part of thelate CMV complex). A slow rise in the $A C$ activity can be seen in both $\mathrm{GO}$ and NOGO immediately after S1, but it extends to the late CMV in the GO condition for some, but not all, of the runs and subjects. Activations of IPF and inferior parietal areas was identified in all subjects, but at varied latencies.

Restricting attention to activations from one subject increased the consistency of slow CM V-like components across runs, as shown in Fig. 3 for one of the lefthanded subjects. Experiment 2, using a full-head biomagnetometer, pursued the single-subject approach, and the introduction of baseline control conditions and repetitions provided a firmer foundation for the analysis. The specificity of regional activations observed in Experiment 1 was confirmed as expected in the findings of Experiment 2 (e.g., Fig. 5a). The wider sensor coverage showed clearly the contributions to the early and late CMV from the IPF and PIP region and revealed activations in new areas, the SMA and PCC. The absence of these activations from the analysis of data from Experiment 1 is not surprising, because of the unfavorable sensor coverage.

The key role of prefrontal cortex in the CMV generation in general and inhibition of movement has been documented in studies with animals (Sasaki et al., 1989) and humans, in studies with normal subjects (Gemba and Sasaki, 1989) as well as in comparisons between normals and patients (Rosahl and Knight, 1995). Evidence for modulation of the CNV by lateral parietal areas was also provided by human studies (Gemba and Sasaki, 1989; Rosahl and Knight, 1995). Animal experiments provide evidence that well-segregated very focal areas are involved: Sasaki and colleagues, for example, identified focal bilateral areas in the principal sulcus of monkey which are preferentially activated by the NOGO stimuli (Sasaki et al., 1989); the electrical stimulation of these areas during the GO condition delivered at the latencies of the peak of "NOGO" activations produced not only movement suppression but also delay in the execution of movement by a few hundreds of milliseconds (Sasaki et al., 1989). In contrast EEG studies from the scalp show widespread potentials in frontal and parietal areas (Gemba and Sasaki, 1989, Rosahl and Knight, 1995). The focal nature of the activations is, however, confirmed in the intracranial studies (I keda et al., 1995, 1996; Hamano et al., 1997; Lamarche et al., 1995). These studies further demonstrated that there are many focal areas which become active when a CNV protocol is used. These studies show also not only that the activation pattern varies between subjects, but also that small changes in the CNV protocol can introducelargechanges in the activations. This last point makes it hard to relate the excellent fMRI study of Konishi et al. (1998) with the rest of the vast literature of human CNV studies. Konishi et al. used $\mathrm{fMRI}$ and a long IS interval which allowed them to bin and to study the GO and NOGO trials separately. Unfortunately the protocol did not include a proper warning stimulus, and the analysis was therefore restricted to the time after the imperative stimulus. Nevertheless the clear finding of a right hemisphere IPF focal activation in the NOGO responses is significant and must be eventually understood together with the results reported in our paper and others' findings. Given these complications we decided to explore how the CMV is generated and how it changes in time, by studying in detail one subject. We had al ready demonstrated in our earlier studies (I oannides et al., 1994; Liu et al., 1996) the feasibility of identifying focal activations from MEG data of a single subject (even in single trial) using MFT. This liberates us from the constraints of assuming focal activations a priori and relies on the data of each time slice to dictate the number and nature of the strongest generators of the observed signal (Taylor et al., 1999).

For the single-subject study the left inferior prefrontal area was consistently activated in all runs, starting soon after S1 with an early CMV peaked at $900 \mathrm{~ms}$ and followed by a late CMV with onset of 1.5-2 s before S2. During the interval between S1 and S2 the early and late CMV-like activation was evident in this area, showing best as the difference between the GO and the NOGO activations. The pattern is consistent with an inhibitory role, as its activity decreases immediately with onset of S2, possibly to allow the execution of the movement. The early activation, coming soon after the auditory- and motor-related areas have completed evoked-like responses to the warning stimulus, is also consistent with a combination of immediate inhibitory function coupled to holding of information regarding theimpending movement (theactivity is much stronger in the GO condition).

The present study shows that during the GO task strong activity is evident in the supplementary motor area and posterior cingulate cortex. This is in contrast to previous MEG/EEG studies which concluded that symmetrical SMA activation should result in a considerable cancellation of their fields (Bötzel et al., 1993; Nagamine et al., 1996). In this study, the bilateral SMA response was identified from the top and the side MFT 
analysis (with 90 sensors each time). These observations agree with findings from studies using implanted intracranial electrodes (I keda et al., 1996; Hamano et al., 1997). Particularly in GO cases the SMA activity increases 200-300 ms after S1, which is well over $3 \mathrm{~s}$ before any movement has to be made, but this is where the movement-planning stage begins. In terms of latency this agrees with our earlier findings (Liu et al., 1996). The new twist added in the present analysis is the lability of the SMA and PCC activations. Even for the highly trained subject J $D$, a repetition of the task leads to the samelatency for the early CMV component, but a marked reduction in amplitude and an almost elimination of the late component. This high lability of the SMA activation is consistent with numerous animal experiments in which SMA plasticity is demonstrated (Tanji, 1994).

In summary, our work adds to the evidence that CMV generators are highly distributed over the human brain, variable from subject to subject, and labile in detail within subjects and that MFT analysis of MEG data can produce very concrete identifications of focal regional activations, including temporal sequencing and a precise statement about which region shows plasticity through modified activations when the experiment is repeated. The specific results reported here apply only to a single subject. Generalizations are suggestive, but they can be made only with confidence after many such studies have been completed. Given the lengthy nature of the experiments and the long analysis such an undertaking is potentially very useful but also formidable.

\section{ACKNOWLEDG MENTS}

The first author gratefully acknowledges the support from RIKEN during his stay at the Laboratory for Human Brain Dynamics, Brain Science Institute (BSI) (RIKEN, J apan), where some of the analysis was completed. Both authors thank Professor Karl Zilles and Dr. Klaus Martin Stephan for critically reading the manuscript. We also thank Dr. Huber Preissl from MEG Center, Institute of Medical Psychology and Behavioural Neurobiology (Germany), as well as Mr. Sixtus Lee and Dr. Douglas Cheyne from CTF System, Inc. (Vancouver, Canada) for their support in doing the experiment.

\section{REFERENCES}

Basile, L. F. H., Rogers, R. L., Bourbon, T. W., and Papanicolaou, A. C. 1994. Slow magnetic flux from human frontal cortex. Electroencephalogr. Clin. Neurophysiol. 90:157-165.

Bötzel, K., Plendl, H., Paulus, W., and Scherg, M. 1993. Bereitschaftspotential: Is there a contribution of the supplementary motor area? Electroencephal ogr. Clin. Neurophysiol . 89:187-196.

Cheyne, D., Vrba, J., Cheung, T., Burbank, M., Weinberg, H., and Lindinger, G. 1995. Source models of slow magnetic fiel ds accompanying movement: Whole cortex measurements using software gradiometers. In Biomagnetism: F undamental Research and Clini- cal Applications (L. Deecke, C. Baumgartner, and G. Stroink, Eds.), pp. 125-130. Elsevier, Amsterdam.

Elbert, T., Rockstroh, B., Hampson, S., Pantev, C., and Hoke, M. 1994. The magnetic counterpart of the contingent negative variation. Electroencephal ogr. Clin. Neurophysiol . 92:262-272.

Gemba, H., and Sasaki, K. 1989. Potential related to no-go reaction of go/no-go hand movement task with color discrimination in human. Neurosci. Lett. 101:263-268.

Hamano, T., Lüders, H. O., I keda, A., Collura, T. F., Comair, Y. G., and Shibasaki, H. 1997. The cortical generators of the contingent negative variation in humans: A study with subdural electrodes. Electroencephalogr. Clin. Neurophysiol . 97:257-268.

Howard, R., and Lumsden, J . 1996. A neurophysiological predictor of reoffending in special hospital patients. Criminal Behav. Mental Health 6:147-156.

I keda, A., Lüders, H. O., Shibasaki, H., Collura, T. F., Burgess, R. C., Moris, H. H., and Hamano, T. 1995. Movement-related potentials associated with bilateral simultaneous and unilateral movements recorded from human supplementary motor area. Electroencephalogr. Clin. Neurophysiol. 95:323-334.

Ikeda, A., Lüders, H. O., Collura, T. F., Burgess, R. C., Moris, H. H., Hamano, T., and Shibasaki, H. 1996. Subdural potentials at orbitofrontal and mesial prefrontal areas accompanying anticipation and decision making in humans: A comparison with Bereitschaftspotential. Electroencephalogr. Clin. Neurophysiol . 98:206212.

I keda, A., Shibasaki, H., Kaji, R., Terada, K., Nagamine, T., Honda, M., and Kimura, J . 1997. Dissociation between contingent negative variation (CNV) and Bereitschaftspotential (BP) in patients with parkinsonism. Electroencephalogr. Clin. Neurophysiol. 102:142151.

Ioannides, A. A., Bolton, J. P. R., and Clarke, C. J. S. 1990. Continuous probabilistic solution to the biomagnetic inverse problem. Inverse Probl . 6:523-542.

I oannides, A. A., Fenwick, P. B. C., Lumsden, J ., Liu, M. J ., Bamidis, P. D., Squires, K. C., Lawson, D., and Fenton, G. W. 1994. Activation sequence of discrete brain areas during cognitive processes: Results from magnetic field tomography. Electroencephalogr. Clin. Neurophysiol. 91:399-402.

Kawashima, R., Satoh, K., Itoh, H., Ono, S., Furumoto, S., Gotoh, R., Koyama, M., Yoshioka, S., Takahashi, T., Takahashi, K., Yanagisawa, T., and Fukuda, H. 1996. Functional anatomy of GO/ NOGO discrimination and response selection-A PET study in man. Brain Res. 728:79-89.

Konishi, S., Nakajima, K., Uchida, I., Sekihara, K., Miyashita, Y. 1998. No-go dominant brain activity in human inferior prefrontal cortex revealed by functional magnetic resonance imaging. Eur. J Neurosci. 10:1209-1213.

Kristeva, R., Cheyne, D., and Deecke, L. 1991. Neuromagnetic fields accompanying unilateral and bilateral voluntary movements: Topography and analysis of cortical sources. Electroencephalogr. Clin. Neurophysiol. 81:284-298.

Lamarche, M., L ouvel, J ., Buser, P., and Rektor, I. 1995. I ntracerebral recordings of slow potentials in a contingent negative variation paradigm: An exploration in epileptic patients. Electroencephalogr. Clin. Neurophysiol . 95:268-276.

Liu, M. J ., Fenwick, P. B. C., Lumsden, J ., Lever, C., Stephan, K. M., and Ioannides, A. A. 1996. Averaged and single-trial analysis of cortical activation sequences in movement preparation, initiation and inhibition. Hum. Brain Mapp. 4:254-264.

Nagamine, T., Kajola, M., Salmelin, R., Shibasaki, H., and Hari, R. 1996. Movement-related sl ow cortical fields and changes of spontaneous MEG- and EEG-brain rhythms. Electroencephalogr. Clin. Neurophysiol. 99:274-286. 
Nakamura, M., Ozawa, N., Shinba, T., and Yamamoto, K. 1992. CNV-like potentials on the cortical surface associated with conditioning in head-restrained rats. Electroencephalogr. Clin. Neurophysiol. 88:155-162.

Rosahl, S. K., and Knight, R. T. 1995. Role of prefrontal cortex in generation of the contingent negative variation. Cereb. Cortex $\mathbf{2}$ 123-134.

Sasaki, K., Gemba, H., and Tsujimoto, T. 1989. Suppression of visually initiated hand movement by simulation of the prefrontal cortex in the monkey. Brain Res. 495:100-107.
Tanji, J . 1994. The supplementary motor area in the cerebral cortex. Neurosci. Res. 19:251-268.

Taylor, J . G., I oannides, A. A., and Müller-Gärtner, H.-W. 1999. IEEE Trans. Med. Imag. 2:151-163.

Walter, W. G., Copper, R., Aldridge, V. J ., McCallum, W. C., and Winter, A. L. 1964. Contingent negative variation: An electric sign of sensori-motor association and expectancy of the human brain. Nature 203:380-384.

Weinberg, H., Brickett, P., Deecke, L., and Boschert, J . 1983. Slow magnetic fields of the brain preceding movements and speech. Nuovo Cimento 2D:495-504. 